\title{
Idarucizumab Reverses Dabigatran Anticoagulant Activity in Healthy Chinese Volunteers: A Pharmacokinetics, Pharmacodynamics, and Safety Study
}

Zining Wang $\cdot$ Xia Zhao $\cdot$ Pengkang He $\cdot$ Shuqing Chen •

Jie Jiang $\cdot$ Akiko Harada $\cdot$ Steven Brooks $\cdot$ Yimin Cui (D)

Received: May 25, 2020 / Published online: July 20, 2020

(C) The Author(s) 2020

\begin{abstract}
Introduction: Idarucizumab is a humanized monoclonal antibody fragment that specifically binds to dabigatran with high affinity and reverses its anticoagulant effect. This study investigated the pharmacokinetics (PK) and pharmacodynamics (PD) of idarucizumab in healthy Chinese subjects at steady state of dabigatran and explored the effect of idarucizumab on PK and PD of dabigatran.

Methods: Twelve subjects received dabigatran etexilate treatment alone $(220 \mathrm{mg}$ twice daily,
\end{abstract}

Zining Wang and Xia Zhao contributed equally to this work.

Digital Features To view digital features for this article go to https://doi.org/10.6084/m9.figshare.12613334.

Z. Wang · X. Zhao · S. Chen · Y. Cui $(\bowtie)$

Department of Pharmacy, Peking University First

Hospital, Beijing, China

e-mail: cui.pharm@pkufh.com; cuiymzy@126.com

P. He · J. Jiang

Department of Cardiology, Peking University First

Hospital, Beijing, China

A. Harada

Clinical PK/PD Department, Nippon Boehringer

Ingelheim Co., Ltd, Kobe, Japan

S. Brooks

Biostatistics and Data Sciences Department,

Boehringer Ingelheim (China) Investment Co., Ltd,

Shanghai, China b.i.d., oral). After a washout period, the 12 subjects again received dabigatran etexilate (220 mg b.i.d., oral) and idarucizumab $(2.5+2.5 \mathrm{~g}$, intravenous) $2 \mathrm{~h}$ after the last administration of dabigatran etexilate.

Results: The geometric mean (gMean) values of area under the plasma concentration-time curve $\left(\mathrm{AUC}_{0-\infty}\right)$ and maximum concentration $\left(C_{\max }\right)$ were $44,200 \mathrm{nmol} \mathrm{h} / \mathrm{L}$ and $30,900 \mathrm{nmol} /$ $\mathrm{L}$, respectively. An amount of $35.3 \mu \mathrm{mol}$ of idarucizumab, corresponding to $33.8 \%$ of the total dose, was excreted by urine over $72 \mathrm{~h}$. The area under the effect $\left(\right.$ AUEC $\left._{a b o v e, 2-12}\right)$ in the presence and absence of idarucizumab was close to zero for all coagulation parameters, diluted thrombin time (dTT), ecarin clotting time (ECT), activated partial thromboplastin time (aPTT), and thrombin time (TT), which indicated the reversal of dabigatran anticoagulation by idarucizumab. There were no serious adverse events reported in this study. No subject tested positive for anti-idarucizumab antibodies.

Conclusion: Idarucizumab was well tolerated and no subject tested positive for anti-idarucizumab antibodies in this study. PK and PD of idarucizumab in healthy Chinese subjects at a steady state of dabigatran were comparable with those in Japanese and Caucasian subjects.

Clinical registration: ClinicalTrials.gov Identifier No. NCT03086356. 
Keywords: Dabigatran etexilate; Healthy Chinese subjects; Idarucizumab; Pharmacokinetic/pharmacodynamic; Safety

\section{Key Summary Points}

Why carry out this study?

The incidence of atrial fibrillation-related stroke has increased over the past 8 years in China as well as the use of oral anticoagulation, e.g., dabigatran etexilate.

As with all anticoagulants, bleeding is the main side effect of dabigatran etexilate, which can be reversed by idarucizumab, but its clinical pharmacology in Chinese subjects is limited.

\section{What was learned from the study?}

In this study, the pharmacokinetics and pharmacodynamics of idarucizumab at a steady state of dabigatran in healthy Chinese subjects were comparable with those in Japanese and Caucasians.

Idarucizumab was also well tolerated and no subjects tested positive for antiidarucizumab antibodies in Chinese subjects.

\section{INTRODUCTION}

The Chinese population has been shown to have a slightly higher overall stroke incidence and a higher proportion of intracerebral hemorrhage than the Caucasian population [1]. The incidence of atrial fibrillation (AF)-related stroke has increased significantly over the past 8 years in China, and there is an increase in oral anticoagulation use in Chinese patients with $\mathrm{AF}$ in recent years [2-5]. Dabigatran etexilate, an oral direct thrombin inhibitor, has been registered worldwide for the prevention of stroke in patients with $\mathrm{AF}$ and for the treatment and secondary prevention of venous thromboembolism [6-10]. In the randomized evaluation of long-term anticoagulation therapy (RE-LY) study, compared with warfarin, dabigatran etexilate demonstrated superior efficacy and similar bleeding rates with $150 \mathrm{mg}$ twice daily (b.i.d.) and non-inferiority and an improved bleeding profile with $110 \mathrm{mg}$ b.i.d.; these benefits were consistent across Asian and non-Asian subgroups $[6,11]$.

As with all anticoagulants, bleeding is the main side effect $[6,12-14]$ and the RE-LY trial reported that major bleeding events were observed in $3.11 \%$ of patients and life-threatening-bleeding events in $1.45 \%$ of patients treated with $150 \mathrm{mg}$ dabigatran etexilate b.i.d. [6], while $2 \%$ of the patients required an urgent surgery or procedure over 2 years [15]. Drug discontinuation and/or transfusion of red cell concentrates could be effective strategies to manage the dabigatran-associated bleeding, and further reversal of this anticoagulant effect could be achieved by the dabigatran-specific reversal agent idarucizumab [16-18]. Reversal strategies such as idarucizumab may also be indicated for anticoagulated patients with overdose when rapid normalization is not expected [19-21].

First approved in the USA in 2015, idarucizumab is a humanized monoclonal mouse antibody fragment (Fab) that binds to dabigatran, thereby reversing its anticoagulant effect [18]. To date, idarucizumab has been approved in more than 55 countries worldwide, including the European Union and Japan. In June 2018, the recommended dose of $5 \mathrm{~g}$, provided as two separate vials each containing $2.5 \mathrm{~g} / 50 \mathrm{~mL}$ idarucizumab, was approved in China [22].

The clinical pharmacology of idarucizumab has been investigated in several phase I studies involving healthy Caucasian and Japanese subjects [23-26]. A phase III study that investigated idarucizumab in dabigatran etexilate-treated patients with uncontrollable or life-threatening bleeding or in need of an emergency surgery or procedure showed that $5 \mathrm{~g}$ of idarucizumab can reverse the anticoagulant effect of dabigatran $[17,27]$. On the basis of the results of these studies, idarucizumab has a low immunogenic potential $[28,29]$. However, to date, the clinical pharmacology data of idarucizumab in Chinese population has been limited. 
The present study aims to investigate the pharmacokinetics (PK) and pharmacodynamics (PD) of idarucizumab in healthy Chinese male and female subjects at a steady state of dabigatran and to explore the effect of idarucizumab on dabigatran's PK and PD.

\section{METHODS}

This trial was conducted between 22 May 2017 and 12 September 2017 at Peking University First Hospital, Beijing, China.

\section{Study Subjects}

Healthy male and female subjects aged 18 to 45 years (body mass index at least 19 and less than $24 \mathrm{~kg} / \mathrm{m}^{2}$ ) were included. Exclusion criteria included any illness or infection, abnormal values for prothrombin time, activated partial thromboplastin time (aPTT), and platelet counts that were considered as safety parameters by the investigator to be clinically relevant.

The study was approved by the Ethics Committee for Clinical Trials, Peking University First Hospital, Beijing, China. All procedures performed in studies involving human participants were in accordance with the ethical standards of the institutional review board and with the 1964 Helsinki Declaration and its later amendments or comparable ethical standards, and in accordance with the principles of Good Clinical Practice and local guidelines. Written informed consent was obtained from all individual participants prior to study enrolment.

\section{Study Design}

This was an open-label, single-center, two-part trial conducted in one group of healthy subjects. Idarucizumab (solution for infusion) and dabigatran etexilate (capsule) were produced and provided by Boehringer Ingelheim Pharma $\mathrm{GmbH} \&$ Co. KG, Biberach/Riss, Germany. During the first part of the treatment period, dabigatran etexilate was administered alone. All subjects received $220 \mathrm{mg}$ dabigatran etexilate b.i.d. for 3 days (days 1-3) and a single $220 \mathrm{mg}$ dose on day 4 . The dose was chosen to achieve the maximum concentration of dabigatran similar to concentrations obtained after administration of dabigatran etexilate $150 \mathrm{mg}$ b.i.d. to the patient population with nonvalvular atrial fibrillation of RE-LY [6]. During the second part of the treatment period, and after a washout period of 3 days (days 5-7), subjects again received dabigatran etexilate b.i.d. for 3 days (days 8-10) and a single 220-mg dose on day 11. Idarucizumab was administered intravenously approximately $2 \mathrm{~h}$ after the last dabigatran etexilate administration in two short infusions of $2.5 \mathrm{~g}$ each within a 15-min interval.

\section{Pharmacokinetics Assessment}

Blood samples for idarucizumab and dabigatran PK analysis and anti-idarucizumab antibodies (ADA) analysis were collected into K3-ethylendiaminetetraacetic acid (EDTA) anticoagulant blood drawing vials, and centrifuged at $2000-4000 \times g$ for $10 \mathrm{~min}$ at $4-8{ }^{\circ} \mathrm{C}$ within $30 \mathrm{~min}$ after the blood samples were taken. The plasma was divided into aliquots before being stored at $-70^{\circ} \mathrm{C}$ or below. Urine samples were taken cumulatively during sampling intervals into a polyethylene container and stored at $4-8{ }^{\circ} \mathrm{C}$ for the remainder of that collection interval, and finally stored at $-70{ }^{\circ} \mathrm{C}$ or below.

\section{Idarucizumab PK Parameters}

Idarucizumab PK parameters $C_{\max }$ (maximum measured concentration in plasma), $\mathrm{AUC}_{0-\infty}$ (area under the concentration-time curve in plasma over the time interval from 0 extrapolated to infinity), and $\mathrm{Ae}_{0-72}$ (amount eliminated in urine over the time interval from 0 to $72 \mathrm{~h}$ ) were assessed as primary endpoints and other PK parameters were calculated appropriately.

$\mathrm{AUC}_{0-\infty}$ was calculated as $\mathrm{AUC}_{0-\infty}=$ $\mathrm{AUC}_{\text {last }}+C_{t} / \lambda z$, where $C_{t}$ is the last measurable concentration. AUC last was calculated using a linear up-log down method. $\lambda z$ was calculated by linear least squares analysis using the last three or more non-zero plasma concentration values. The apparent terminal rate constant $\lambda z$ 
will be estimated from a regression of $\ln C$ versus time over the terminal log-linear drug disposition portion of the concentration-time profiles.

For idarucizumab PK, plasma samples were collected pre-dose and post dose at $2,10,30$ min and 1, 1.5, 2, 3, 4, 6, 8, 12, 24, 48, 72, $96 \mathrm{~h}$. All urine voided between $0-12$ and $12-24 \mathrm{~h}$ post dose was collected.

The idarucizumab concentrations in plasma and urine were determined by a validated enzyme-linked immunosorbent assay (ELISA) methods at Covance Laboratories, Inc., Chantilly, Virginia, USA. The method began by coating a microtiter plate with a mouse monoclonal anti-BI 655075 antibody (clone 5H4). After an overnight incubation, the plates were blocked, washed, and incubated with diluted calibrators, quality controls, and samples which were previously pre-treated with dabigatran. Bound analyte was detected with anti-human immunoglobulin $\mathrm{G}$ horseradish peroxidase (IgG HRP). Tetramethylbenzidine (TMB) substrate was then added, and plates were read calorimetrically on a plate reader. The results were analyzed with a five-parameter logistic fit of the calibration standard curve.

\section{Dabigatran PK Parameters}

Dabigatran is known to be conjugated with glucuronic acid to yield pharmacologically active acylglucuronides. Total dabigatran (unconjugated plus glucuronide-conjugated dabigatran) and unbound dabigatran (total dabigatran neither bound to idarucizumab nor to plasma proteins) were measured in plasma. Dabigatran PK parameters $A_{0-74, s s}$ of total dabigatran (eliminated in urine over the time interval from 0 to $74 \mathrm{~h}$ at a steady state) and $\mathrm{AUC}_{2-12, \mathrm{ss}}$ of unbound dabigatran (in plasma over the time interval from 2 to $12 \mathrm{~h}$ at a steady state) on day 4 and day 11 were assessed as secondary endpoints and other PK parameters were calculated appropriately.

Dabigatran concentrations in plasma and urine were determined by validated high-performance liquid chromatography-tandem mass spectrometry (HPLC-MS/MS) assays at Nuvisan
GmbH, Neu-Ulm, Germany. In urine, the concentration of unconjugated and total dabigatran were measured. The validated assay methods involved direct injection on a precolumn and subsequent transfer by online column switching onto the analytical RP18 column. For quantification of dabigatran, the following ions were recorded: $472 \rightarrow 289 \mathrm{amu}$ (analyte) and $478 \rightarrow 295 \mathrm{amu}$ (isotope-labelled internal standard). In addition, ions $648 \rightarrow 289$ amu were monitored for dabigatran acylglucuronides.

\section{Anti-idarucizumab Antibodies}

For the analysis of anti-idarucizumab antibodies (ADAs), samples were collected at baseline, end of study (EOS), and 4-week and 3-month followups. ADA in plasma was determined by a validated drug-bridging electrochemiluminescence (ECL) method at Covance Laboratories, Inc., Chantilly, Virginia, USA. Acidified samples were added to a mixture which contained Tris base, biotin-labeled idarucizumab, and Sulfo-TAG-labeled idarucizumab and allowed to complex with ADA. The labeled drug-ADA complexes were transferred to a streptavidin plate where they were captured. The plate was washed to remove any nonspecific bound complexes, and read buffer $(1 \times)$ was added to each well of the plate. The plate was read on the Meso Scale Discovery (MSD) Sector ${ }^{\mathrm{TM}}$ Imager 6000.

\section{Pharmacodynamics Assessment}

The anticoagulant effect of dabigatran and its reversal by idarucizumab were assessed by measuring the blood coagulation parameters, diluted thrombin time (dTT), thrombin time (TT), aPTT, and ecarin clotting time (ECT). The blood samples were collected in citrated plasma collection tubes and centrifuged immediately at $2500 \times g$ for $20 \mathrm{~min}$ at $4{ }^{\circ} \mathrm{C}$. Plasma was divided into six separate polypropylene vials $(2 \times 100 \mu \mathrm{L}$ for dTT, $2 \times 150 \mu \mathrm{L}$ for TT determination, $2 \times 300 \mu \mathrm{L}$ for aPTT and ECT) and frozen immediately at $-20^{\circ} \mathrm{C}$ or below. For dTT, the parameter AUEC ${ }_{\text {above,2-12 }}$ (area under the curve from 2 to $12 \mathrm{~h}$ under 
consideration of the baseline value) was determined on day 4 (dabigatran etexilate only) and on day 11 (dabigatran etexilate and idarucizumab).

In citrated plasma samples, dTT (Hemoclot, Hyphen BioMed, Neuville sur Oise, France), ECT (in-house assay, $6 \mathrm{U} / \mathrm{mL}$ ecarin; Pentapharm, Basel, Switzerland), TT (STA Thrombin Reagent, Roche Diagnostics $\mathrm{GmbH}$, Mannheim, Germany), and aPTT (CK Prest, Diagnostica Stago, Asnières-sur-Seine, France) were determined by validated assays according to the manufacturer's instructions at Menal GmbH (Emmendingen, Germany) [30].

\section{Safety Assessment}

All adverse events (AEs) which occurred during the course of the study were recorded and coded using the Medical Dictionary for Drug Regulatory Affairs (MedDRA, version 20.0). The frequency of AEs, using incidence rates, was summarized by relationship to study drug, severity, system organ class, and preferred term. Safety was also evaluated on the basis of safety laboratory tests (including hematology, coagulation parameters, clinical chemistry, and urinalysis), physical examination, vital signs, electrocardiogram (ECG), pO2 monitoring, local tolerability, and ADA.

\section{Statistical Analysis}

The PK, PD, and safety analyses were all descriptive. For all parameters (dTT, TT, aPTT, and ECT), reversal to baseline was defined as a coagulation test value returning below the upper limit of normal (ULN) as determined based on the pre-dose values of the coagulation parameter before dabigatran drug administration (primary analysis). The definition of ULN was based on the pre-dose PD measurements plus an appropriate tolerance (two standard deviations, SDs) for the respective PD measurements.

The safety and PK analyses included all subjects entered. For safety evaluation (except for ECG), the baseline was defined as the last available measurement prior to the first dabigatran etexilate administration.

All analyses were performed using SAS $^{\circledR}$ version 9.4. PK and PD parameters were calculated using WinNonlin ${ }^{\mathrm{TM}}$ software (professional Network version 6.3, Pharsight Corporation, Mountain View, CA 94041-1530, USA).

\section{RESULTS}

\section{Subjects}

A total of 12 subjects (six male and six female) were entered into the trial and received dabigatran etexilate and idarucizumab as planned without any important protocol violations.

The mean age, height, weight, and BMI were $35.6(\mathrm{SD} 5.8)$ years, $164.2(\mathrm{SD} 9.7) \mathrm{cm}, 59.08$ (SD $6.81) \mathrm{kg}$, and 21.88 (SD 0.94$) \mathrm{kg} / \mathrm{m}^{2}$, respectively. The majority of the subjects were nonsmokers (10 subjects [83.3\%]) and non-drinkers (9 subjects [75.0\%]). Four subjects (33.3\%) were reported with concomitant diagnoses which were no longer present at the time of screening. In all cases, the reported diagnoses were not considered to interfere with the analyses performed in this trial. Only one subject (8.3\%) was reported with concomitant therapy with mupirocin ointment to treat a bruise, which was considered to not interfere with the analyses performed in this trial.

\section{Pharmacokinetics}

\section{Idarucizumab PK parameters}

As shown in Fig. 1, the mean idarucizumab concentration in plasma peaked at around the end of infusion with a median $t_{\max }$ of $0.450 \mathrm{~h}$ and declined rapidly with a gMean initial halflife $\left(t_{1 / 2,2}\right)$ of $0.758 \mathrm{~h}$ and a terminal elimination half-life $\left(t_{1 / 2}\right)$ of $8.18 \mathrm{~h}$ after administration of $2.5+2.5 \mathrm{~g}$ idarucizumab (Table 1 ). The gMean values of $\mathrm{AUC}_{0-\infty}$ and $C_{\max }$ in plasma were $44,200 \mathrm{nmol} \mathrm{h} / \mathrm{L}$ and $30,900 \mathrm{nmol} / \mathrm{L}$, respectively. The gMean values of clearance (CL) and the apparent volume of distribution at steady state $\left(V_{\text {ss }}\right)$ were $39.4 \mathrm{~mL} / \mathrm{min}$ and $6.47 \mathrm{~L}$, respectively. 


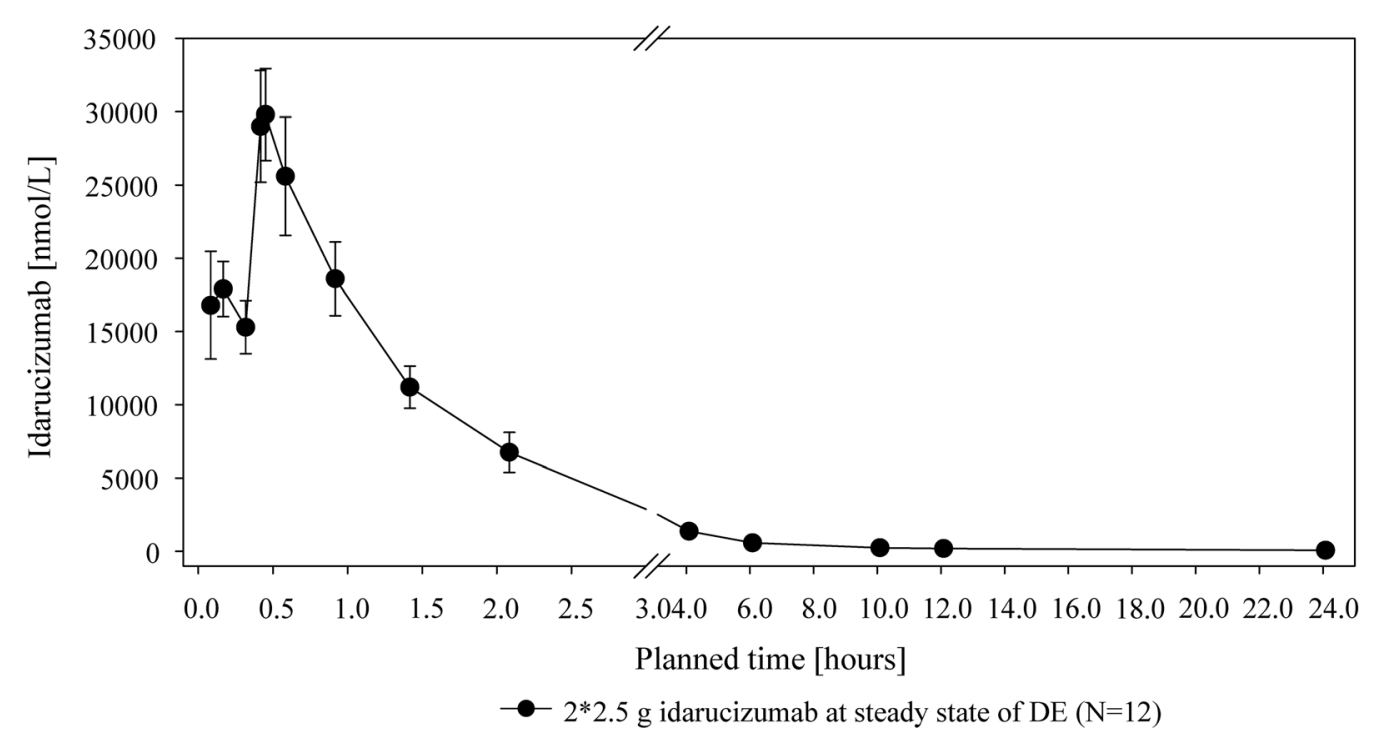

Fig. 1 Plasma concentration-time profile of idarucizumab at steady state of DE. DE dabigatran etexilate

Table 1 Summary of PK parameters of idarucizumab

\begin{tabular}{llll}
\hline DE $(\mathbf{2 2 0} \mathbf{~ m g})+$ idarucizumab $(\mathbf{2 . 5}+\mathbf{2 . 5} \mathbf{g})$ & Number & gMean & gCV (\%) \\
\hline $\mathrm{AUC}_{0-\infty}[\mathrm{nmol} \mathrm{h} / \mathrm{L}]$ & 12 & 44,200 & 10.1 \\
$C_{\max }[\mathrm{nmol} / \mathrm{L}]$ & 12 & 30,900 & 9.86 \\
$t_{\max }[\mathrm{h}]^{\mathrm{a}}$ & 12 & 0.450 & $0.417-0.517$ \\
$t_{1 / 2}[\mathrm{~h}]$ & 12 & 8.18 & 17.9 \\
$t_{1 / 2,2}[\mathrm{~h}]$ & 12 & 0.758 & 18.3 \\
$\mathrm{Ae}_{0-72}[\mu \mathrm{mol}]$ & 12 & 35.3 & 57.1 \\
$\mathrm{fe}_{0-72}[\%]$ & 12 & 33.8 & 57.1 \\
$\mathrm{CL}[\mathrm{mL} / \mathrm{min}]$ & 12 & 39.4 & 10.1 \\
$V_{\mathrm{ss}}[\mathrm{L}]$ & 12 & 6.47 & 13.1 \\
\hline
\end{tabular}

${ }^{a}$ Median (min-max)

$D E$ dabigatran etexilate, $A U C$ area under the plasma concentration-time curve, $C_{\max }$ maximum concentration, $g C V$ geometric coefficient of variation, gMean geometric mean, $t_{\max }$ time to maximum plasma concentration, $t_{1 / 2}$ terminal halflife, $t_{1 / 2,2}$ initial half-life, $A e$ amount excreted in urine, $f e$ fraction excreted in urine, $C L$ clearance, $V_{s s}$ volume of distribution at steady state

The largest amount of idarucizumab was excreted in the first urine collection interval $(0-4 \mathrm{~h}, 33.6 \%$ of dose [fe] $)$ and the contribution of subsequent fractions was less than $0.2 \%$ of idarucizumab dose excreted in urine, resulting in $\mathrm{Fe}_{0-72}$ of $33.8 \%$ up to $72 \mathrm{~h}$ (Table 1 ).
No apparent difference was observed for PK of idarucizumab in male and female subjects.

\section{Dabigatran PK Parameters}

At a steady state of dabigatran in the absence of idarucizumab, plasma concentrations of unbound dabigatran reached a peak at $2.5 \mathrm{~h}$ and 
then declined in a bi-exponential manner. Immediately after administration of the first vial of idarucizumab, the mean plasma concentration of unbound dabigatran decreased to below the limit of quantification (BLQ). Subsequently, unbound dabigatran was detectable again between 26 and $74 \mathrm{~h}$ after the last administration of dabigatran etexilate; however, mean concentrations remained below $20 \mathrm{ng} / \mathrm{mL}$ corresponding to $42.4 \mathrm{nmol}$, i.e., a threshold at which little or no relevant anticoagulant effect would be expected [17]. Exposure to unbound dabigatran $\left(\mathrm{AUC}_{2-12, \mathrm{ss}}\right)$ decreased to less than $1 \%$ after administration of idarucizumab from $1270 \mathrm{ng} \mathrm{h} / \mathrm{mL}$ in the absence of idarucizumab to $11.6 \mathrm{ng} \mathrm{h} / \mathrm{mL}$ in the presence of idarucizumab. In contrast, administration of idarucizumab resulted in a rapid, approximately fourfold increase of the mean concentration of total dabigatran, which was inactive because of binding to idarucizumab.

The time profile of idarucizumab, total dabigatran, and unbound dabigatran after administration of $2.5+2.5 \mathrm{~g}$ idarucizumab is presented in Fig. 2. Generally, as long as the concentration of idarucizumab was higher than that of total dabigatran, the unbound dabigatran concentration was BLQ. Unbound dabigatran gradually increased after total dabigatran concentration exceeded idarucizumab concentration. Urinary excretion of total dabigatran temporarily decreased in the presence of idarucizumab $\left(\mathrm{Fe}_{0-12, \mathrm{ss}}: 5.1 \%\right.$ vs $3.2 \%$, at $12 \mathrm{~h}$ ); however, both reached a similar range of approximately $7 \%$ at $74 \mathrm{~h}$, accounting for $11.7 \mathrm{mg}$ and $11.0 \mathrm{mg}$ on day 4 and day 11 , respectively (Fig. 3).

\section{Pharmacodynamics}

The prolongation of the coagulation times dTT, ECT, aPTT, and TT were associated with unbound dabigatran concentrations, which peaked at $2.5 \mathrm{~h}$ after administration of $220 \mathrm{mg}$ dabigatran etexilate, and then decreased close to ULN at $74 \mathrm{~h}$ according to the normal clearance of dabigatran from plasma in the absence of idarucizumab (Fig. 4).

In contrast, immediate and complete reversal was observed after administration of $2.5+2.5 \mathrm{~g}$ idarucizumab in coagulation times and mean coagulation times remained below ULN.

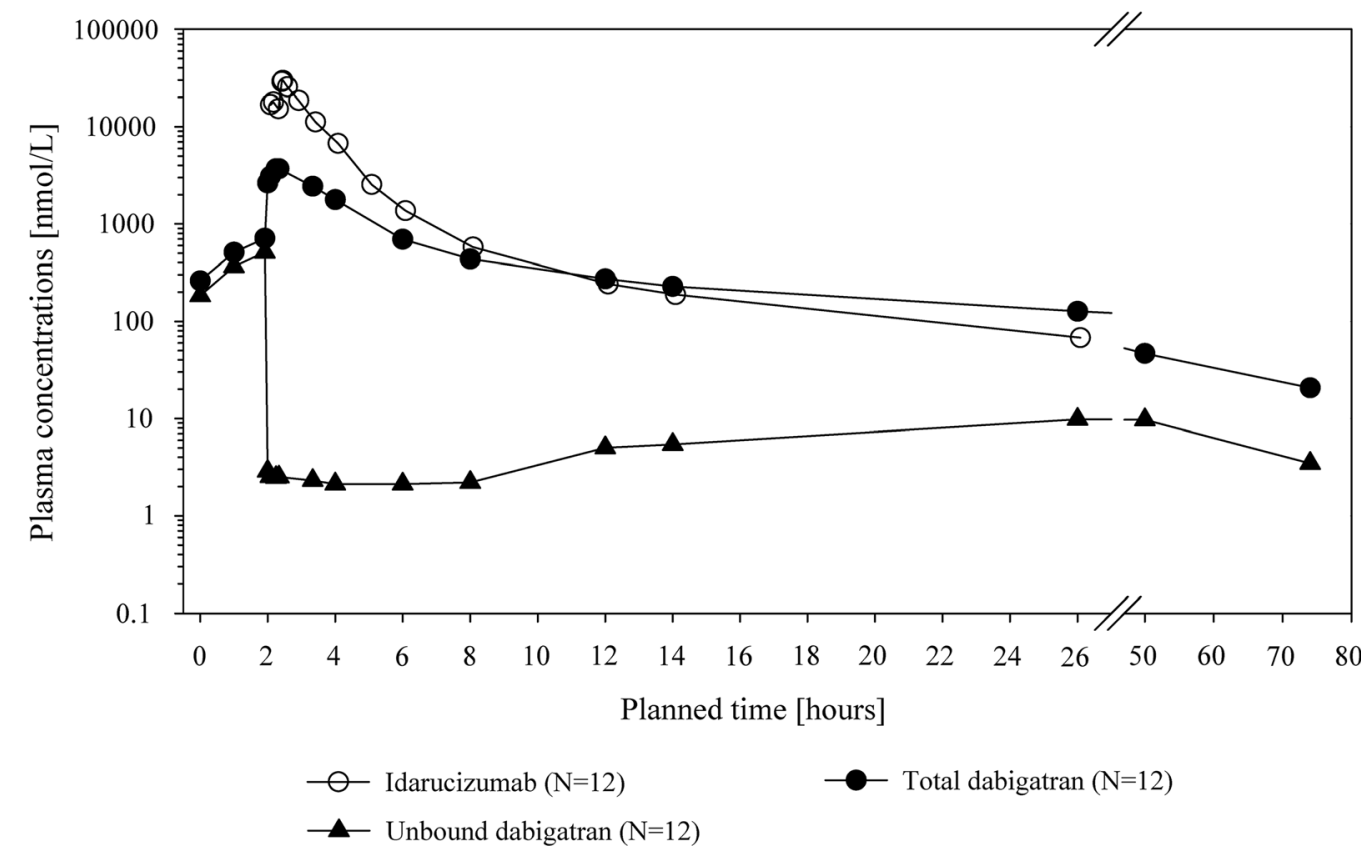

Fig. 2 Plasma concentration-time profile of total dabigatran, unbound dabigatran, and idarucizumab 


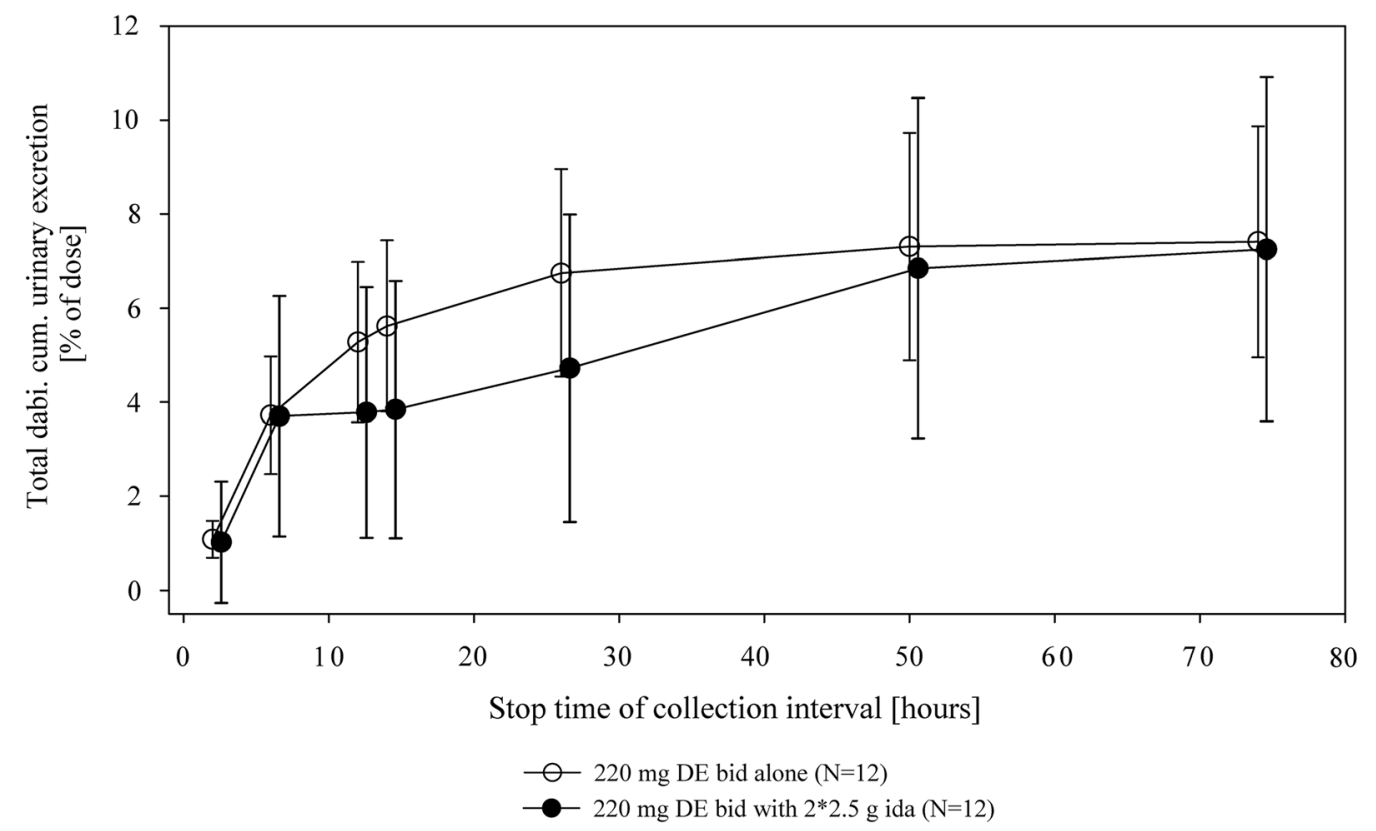

Fig. 3 Time profile of urinary excretion for total dabigatran. DE dabigatran etexilate, ida idarucizumab
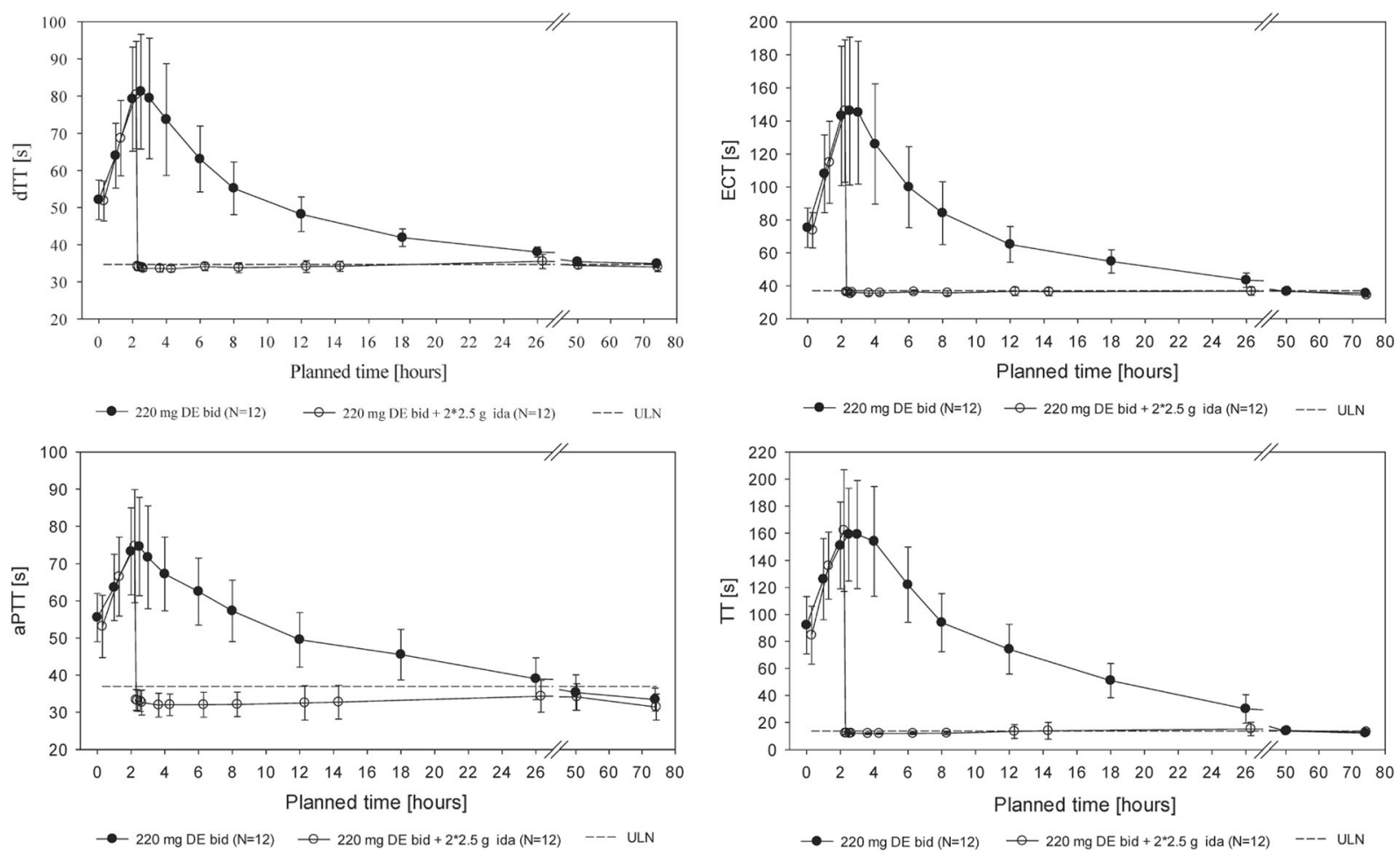

Fig. 4 Effect of idarucizumab $(2.5+2.5 \mathrm{~g})$ on a diluted thrombin time (dTT), b ecarin clotting time (ECT), c activated partial thromboplastin time (aPTT), and variables are overlaid with (day 11) or without idarucizumab (day 4). DE dabigatran etexilate, ida idarucizumab d thrombin time (TT). Effect time profiles of coagulation 
The reduction of coagulation times in response to idarucizumab administration was further demonstrated by calculating the ratio of AUEC $_{\text {above,2-12 }}$ in the presence of idarucizumab and the AUEC ${ }_{\text {above,2-12 }}$ in the absence of idarucizumab. As shown in Table 2, values of AUEC $_{\text {above,2-12 }}$ decreased by greater than or equal to $98 \%$ in the presence of idarucizumab compared with those in the absence of idarucizumab for all coagulation parameters dTT, ECT, aPTT, and TT.

No apparent difference was observed between genders for the reversal demonstrated by coagulation markers.

\section{Safety}

All 12 subjects treated in this trial reported at least one $\mathrm{AE}$. On the basis of the $\mathrm{AE}$ onset, the most frequent AEs between the first intake of dabigatran etexilate until the first infusion of idarucizumab were aPTT prolonged (10 subjects) and prothrombin level decreased (4 subjects). None of these AEs were assessed as drugrelated by the investigator and none required any therapy. The most frequent AEs with an onset between the first infusion of idarucizumab until the end of treatment visit were alterations in urine. This included increased albumin and $\alpha 1$-microglobulin (both for all 12 subjects), proteinuria (11 subjects), and an abnormal urine electrophoresis (10 subjects). All these AEs were assessed as drug-related by the investigator. All drug-related AEs were of mild intensity and did not require therapy. No other significant AEs were recorded (according to ICH E3). No AEs led to discontinuation of the trial drug, and no pre-specified AEs of special interest (AESIs) were reported. No subject had a severe AE; all AEs were of mild intensity. There were no deaths or other serious adverse events (SAEs) reported in this trial.

Descriptive statistics of laboratory parameters did not reveal any relevant changes from baseline except for aPTT, urine $\alpha 1$-microglobulin, urine albumin, and urine IgG. Mean aPTT was transiently prolonged compared with the baseline value on day 4 and again on day 11, i.e., after the subjects had received dabigatran etexilate on the three preceding days. For the parameters urine $\alpha 1$-microglobulin, urine albumin, and urine IgG, a transient increase of the mean values from baseline was observed starting after administration of the first idarucizumab infusion (at day 11, i.e., $242 \mathrm{~h} 15 \mathrm{~min}$ ). Mean values for urine $\alpha 1$-microglobulin, urine albumin, and urine IgG returned to baseline levels on the following day. However, individual subjects also had slightly elevated levels of urine $\alpha 1$-microglobulin or urine $\operatorname{IgG}$ at later time points. There were no clinically relevant findings with respect to vital signs. Local tolerability of the infusion was good.

No subject tested positive for ADA at any time point up to 3 months after idarucizumab administration.

\section{DISCUSSION}

This study investigated the PK and PD of idarucizumab in healthy Chinese male and female subjects for the first time. Consistent with the previous studies in Caucasian and Japanese subjects, immediate and complete reversal was observed in Chinese subjects after administration of $2.5+2.5 \mathrm{~g}$ idarucizumab at the steady state of dabigatran. This was sustained over the entire observation period of $72 \mathrm{~h}$ [23-25]. Idarucizumab was well tolerated by Chinese subjects.

Table 2 Mean ratio for the coagulation measurements dTT, ECT, aPTT, and TT

\begin{tabular}{|c|c|c|c|c|}
\hline Ratio $(N=12,12,12,12)$ & $\mathrm{dTT}$ & ECT & aPTT & TT \\
\hline$\frac{\text { AUEC }_{\text {above }, 2-12} \text { with idarucizumab }}{\text { AUEC }_{\text {above }, 2-12} \text { without idarucizumab }}[S D]$ & $0.01[0.017]$ & $0.02[0.017]$ & $0.02[0.034]$ & $0.00[0.007]$ \\
\hline
\end{tabular}

$S D$ standard deviation, $A U E C$ area under the effect curve 
The overall PK profile of idarucizumab was similar among Chinese, Japanese, and Caucasian subjects [23-25]. However, urinary excretion was slightly higher in Chinese subjects compared with Japanese subjects, while other parameters were similar to those of Japanese subjects. Taking into consideration the high interindividual variability in Chinese subjects as well as the overlap of individual values between Chinese and Japanese subjects, the difference is not considered to be clinically relevant.

Exposure to total dabigatran was comparable between Chinese and Japanese subjects when only male subjects were compared. Of note, exposure was approximately $20 \%$ higher in female subjects than in male subjects in this study, which is in line with known PK characteristics of dabigatran [31].

Peak idarucizumab was achieved at the end of infusion, and resulted in a rapid reduction of unbound dabigatran in plasma. Immediate, complete, and sustained reversal was also demonstrated with $5 \mathrm{~g}$ of idarucizumab in Japanese and Caucasian healthy volunteers $[24,25]$. Therefore, it is concluded that there is no meaningful difference in PD of idarucizumab in Chinese subjects compared with Caucasian and Japanese subjects. Consistent with other countries, the dose of $5 \mathrm{~g}$ was also approved in China.

Overall, idarucizumab and dabigatran etexilate were well tolerated by Chinese subjects in this trial. During treatment with dabigatran etexilate only, the most frequently reported AEs were related to anticoagulation. Accordingly, analysis of laboratory parameters revealed a transient prolongation of aPTT after the subjects had received dabigatran etexilate. This prolongation of aPTT may indicate overcoagulation, and the reported AEs of coagulation parameters were consistent with the mechanism of action of dabigatran etexilate as anticoagulant, and none of them were assessed as idarucizumab-related by the investigator. Increase of urinary protein after idarucizumab administration was considered to be drug-related. According to the reported AEs, the analysis of laboratory parameters also revealed a transient increase in urinary proteins after administration of the first idarucizumab infusion. This has also been observed in the previous phase I trials and likely indicates saturation of the tubular uptake processes for the reabsorption of small proteins from the filtrate [32]. The most frequent AEs for idarucizumab-treated subjects in the previous phase I trials were headache, nasopharyngitis, back pain, and skin irritation, none of which were reported in this trial [23]. There were no clinically relevant findings with respect to vital signs. Local tolerability of the infusion was good. Idarucizumab was not immunogenic in this trial.

Limitations of this study include the relatively limited number of subjects assessed and the fact that only healthy subjects were included. A phase III study (ClinicalTrials.gov NCT03343704) in Chinese subjects is currently ongoing to assess the effect of idarucizumab at a dose of $5 \mathrm{~g}$ on the anticoagulant effect of dabigatran in patients who have either uncontrolled bleeding requiring urgent medical intervention or who require emergency surgery or procedures necessitating rapid reversal of the anticoagulant effect of dabigatran.

\section{CONCLUSION}

Administration of idarucizumab (two intravenous administrations of $2.5 \mathrm{~g}$ each, with a 15-min interval) after dabigatran dosing to a steady state resulted in immediate, complete, and sustained reversal of dabigatran-induced anticoagulant effect in healthy Chinese subjects. The overall PK and PD of idarucizumab in Chinese subjects in this study were similar to those in Japanese and Caucasian subjects. In Chinese subjects, idarucizumab was well tolerated and no subject tested positive for antiidarucizumab antibodies.

\section{ACKNOWLEDGEMENTS}

Boehringer Ingelheim (China) Investment Co., Ltd. was given the opportunity to review the manuscript content for medical and scientific accuracy and intellectual property considerations. The authors wish to thank Yu Wang for 
her statistical contributions. The authors also thank the participants of the study.

Funding. The study and the Rapid Service fee for this manuscript were funded by Boehringer Ingelheim (China) Investment Co., Ltd.

Authorship. All named authors meet the International Committee of Medical Journal Editors (ICMJE) criteria for authorship for this article, take responsibility for the integrity of the work as a whole, and have given their approval for this version to be published.

Medical Writing and Editorial Assistance. Medical writing assistance was provided by Ningning Dong of Boehringer Ingelheim (China) Investment Co., Ltd.

Disclosures. Zining Wang, Xia Zhao, Pengkang He, Shuqing Chen, Jie Jiang and Yimin Cui have nothing to disclosure, Akiko Harada and Steven Brooks are employees of Boehringer Ingelheim.

Compliance with Ethical Guidelines. The study was approved by the Ethics Committee for Clinical Trials, Peking University First Hospital, Beijing, China. All procedures performed in studies involving human participants were in accordance with the ethical standards of the institutional review board and with the 1964 Helsinki Declaration and its later amendments or comparable ethical standards, and in accordance with the principles of Good Clinical Practice and local guidelines. Written informed consent was obtained from all individual participants prior to study enrolment.

Data Availability. Boehringer Ingelheim is committed to responsible sharing of redacted clinical study reports, related clinical documents, and de-identified patient-level clinical study data after drug approval or after termination of the drug development program. The datasets generated for this study can be accessed and analyzed via application through https:// www.vivli.org/.
Open Access. This article is licensed under a Creative Commons Attribution-NonCommercial 4.0 International License, which permits any non-commercial use, sharing, adaptation, distribution and reproduction in any medium or format, as long as you give appropriate credit to the original author(s) and the source, provide a link to the Creative Commons licence, and indicate if changes were made. The images or other third party material in this article are included in the article's Creative Commons licence, unless indicated otherwise in a credit line to the material. If material is not included in the article's Creative Commons licence and your intended use is not permitted by statutory regulation or exceeds the permitted use, you will need to obtain permission directly from the copyright holder. To view a copy of this licence, visit http://creativecommons.org/licenses/by$\mathrm{nc} / 4.0 /$.

\section{REFERENCES}

1. Tsai C-F, Thomas B, Sudlow CL. Epidemiology of stroke and its subtypes in Chinese vs white populations: a systematic review. Neurology. 2013;81: 264-72.

2. Guo Y, Tian Y, Wang H, Si Q, Wang Y, Lip GY. Prevalence, incidence, and lifetime risk of atrial fibrillation in China: new insights into the global burden of atrial fibrillation. Chest. 2015;147: 109-19.

3. Chang S-S, Dong J-Z, Ma C-S, et al. Current status and time trends of oral anticoagulation use among Chinese patients with nonvalvular atrial fibrillation: the Chinese atrial fibrillation registry study. Stroke. 2016;47:1803-10.

4. Wang-Longde LJ, Yi Y, Peng B, Wang Y. The prevention and treatment of stroke still face huge challenges-brief report on stroke prevention and treatment in China, 2018. Chin Circ J. 2019;34: 105-19.

5. Ma C, Li Q. GW28-e1105 Changes in oral anticoagulation treatment after dabigatran availability in China: the GLORIA-AF Registry Program. J Am Coll Cardiol. 2017;70(16 Supplement):C53.

6. Connolly SJ, Ezekowitz MD, Yusuf S, et al. Dabigatran versus warfarin in patients with atrial fibrillation. N Engl J Med. 2009;361:1139-51. 
7. Schulman S, Kearon C, Kakkar AK, et al. Dabigatran versus warfarin in the treatment of acute venous thromboembolism. N Engl J Med. 2009;361: 2342-52.

8. Schulman S, Kakkar AK, Goldhaber SZ, et al. Treatment of acute venous thromboembolism with dabigatran or warfarin and pooled analysis. Circulation. 2014;129:764-72.

9. Connolly SJ, Ezekowitz MD, Yusuf S, Reilly PA, Wallentin L. Newly identified events in the RE-LY trial. N Engl J Med. 2010;363:1875-6.

10. Schulman S, Kearon C, Kakkar AK, et al. Extended use of dabigatran, warfarin, or placebo in venous thromboembolism. N Engl J Med. 2013;368: 709-18.

11. Hori M, Connolly SJ, Zhu J, et al. Dabigatran versus warfarin: effects on ischemic and hemorrhagic strokes and bleeding in Asians and non-Asians with atrial fibrillation. Stroke. 2013;44:1891-6.

12. Granger $\mathrm{CB}$, Alexander JH, McMurray JJ, et al. Apixaban versus warfarin in patients with atrial fibrillation. N Engl J Med. 2011;365:981-92.

13. Patel MR, Mahaffey KW, Garg J, et al. Rivaroxaban versus warfarin in nonvalvular atrial fibrillation. N Engl J Med. 2011;365:883-91.

14. Giugliano RP, Ruff CT, Braunwald E, et al. Edoxaban versus warfarin in patients with atrial fibrillation. N Engl J Med. 2013;369:2093-104.

15. Douketis JD, Healey JS, Brueckmann M, et al. Urgent surgery or procedures in patients taking dabigatran or warfarin: analysis of perioperative outcomes from the RE-LY trial. Thromb Res. 2016;139:77-81.

16. Majeed A, Hwang H-G, Connolly S. Management and outcomes of major bleeding during treatment with dabigatran or warfarin. J Vasc Surg. 2014;59: 871-2.

17. Pollack CV Jr, Reilly PA, Eikelboom J, et al. Idarucizumab for dabigatran reversal. $\mathrm{N}$ Engl J Med. 2015;373:511-20.

18. Eikelboom JW, Quinlan DJ, van Ryn J, Weitz JI. Idarucizumab: the antidote for reversal of dabigatran. Circulation. 2015;132:2412-22.

19. Steffel J, Verhamme P, Potpara TS, et al. The 2018 European Heart Rhythm Association Practical Guide on the use of non-vitamin K antagonist oral anticoagulants in patients with atrial fibrillation: executive summary. Eur Heart J. 2018;2:1231-42.
20. Baugh CW, Levine M, Cornutt D, et al. Anticoagulant reversal strategies in the emergency department setting: recommendations of a multidisciplinary expert panel. Ann Emerg Med. 2019. https://doi.org/10.1016/j.annemergmed. 2019.09.001.

21. Expert Committee of Stroke Prevention ProjectNational Health Commission of the People's Republic of Chinese, Chinese Society of Pacing and Electrophysiology Expert Committee, Chinese College Society of Arrhythmia. Expert consensus on clinical application of idarucizimab-dabigatran specific antagonist. Chin J Cardiac Arrhyth. 2020;24:113-22.

22. China National Medical Products Administration. Idarucizumab injection, S20180012 86980023000365 Boehringer Ingelheim International GmbH [Internet]. 2020. Available from: http://app1.nmpa.gov.cn/. Accessed 31 Jan 2020.

23. Glund S, Moschetti V, Norris S, et al. A randomised study in healthy volunteers to investigate the safety, tolerability and pharmacokinetics of idarucizumab, a specific antidote to dabigatran. Thromb Haemost. 2015;114:943-51.

24. Glund S, Stangier J, Schmohl M, et al. Safety, tolerability, and efficacy of idarucizumab for the reversal of the anticoagulant effect of dabigatran in healthy male volunteers: a randomised, placebocontrolled, double-blind phase 1 trial. Lancet. 2015;386:680-90.

25. Yasaka M, Ikushima I, Harada A, et al. Safety, pharmacokinetics and pharmacodynamics of idarucizumab, a specific dabigatran reversal agent in healthy Japanese volunteers: a randomized study. Res Pract Thromb Haemost. 2017;1:202-15.

26. Glund S, Stangier J, van Ryn J, et al. Effect of age and renal function on idarucizumab pharmacokinetics and idarucizumab-mediated reversal of dabigatran anticoagulant activity in a randomized, double-blind, crossover phase Ib study. Clin Pharmacokinet. 2017;56:41-544.

27. Pollack CV Jr, Reilly PA, Bernstein R, et al. Design and rationale for RE-VERSE AD: a phase 3 study of idarucizumab, a specific reversal agent for dabigatran. Thromb Haemost. 2015;114:198-205.

28. Norris S, Ramael S, Ikushima I, et al. Evaluation of the immunogenicity of the dabigatran reversal agent idarucizumab during phase I studies. Br J Clin Pharmacol. 2017;83:1815-25.

29. Glund S, Coble K, Gansser D, et al. Pharmacokinetics of idarucizumab and its target dabigatran in patients requiring urgent reversal of the 
anticoagulant effect of dabigatran. J Thromb Haemost. 2019;17(8):1319-28.

30. Stangier J, Feuring M. Using the HEMOCLOT direct thrombin inhibitor assay to determine plasma concentrations of dabigatran. Blood Coagul Fibrinol. 2012;23:138-43.

31. Stangier J. Clinical pharmacokinetics and pharmacodynamics of the oral direct thrombin inhibitor dabigatran etexilate. Clin Pharmacokinet. 2008;47: 285-95.

32. Reilly PA, van Ryn J, Grottke O, Glund S, Stangier J. Idarucizumab, a specific reversal agent for dabigatran: mode of action, pharmacokinetics and pharmacodynamics, and safety and efficacy in phase 1 subjects. Am J Med. 2016;129:S64-S72. 